\title{
Left Heart Catheterization with Special Reference to the Transseptal Method
}

\author{
P. G. F. NIXON AND H. IKRAM \\ From the Cardiac Department, Charing Cross Hospital, London W.C.2
}

In the early nineteen-fifties the emergence of cardiac surgery called for accurate diagnosis, and it became necessary to catheterize the left side of the heart. The left atrium was entered by needles inserted through the tracheal wall, the suprasternal notch, and the back of the chest; and the left ventricle was entered by needle puncture, or by the passage of a catheter from the left atrium or the aorta (Lancet, 1957). These methods had diagnostic limitations or disadvantages, and dangers that restricted their use to hospitals where thoracic surgeons were available to deal with the complications (P. Wood, 1961, personal communication).

In 1959 John Ross jun. described atrial septal puncture as a means of entering the left side of the heart, and early in 1960 one of us reported a series of 57 patients (Nixon, 1960). This series has now been extended to 508 , and the results suggest that the technique may be safe enough to be performed with right heart catheterization in the physician's catheter laboratory.

Nearly every patient investigated was a candidate for the surgical treatment of his heart disease, and the need for an accurate estimate of the morbid physiological changes justified a small risk when the alternative course was to operate, or to withhold surgical treatment, on the basis of a diagnosis that was something less than exact. The majority of the patients had mitral valvar disease, and the number investigated reflects the inaccuracy of the traditional methods of diagnosing the various syndromes of obstruction and incompetence. Percutaneous puncture of the left ventricle and/or percutaneous catheterization of the aorta was performed in addition to the atrial septal puncture when the need arose. The purpose of this communication is to describe our experience of the three techniques and

Received November 4, 1965. to consider particularly the features concerned with their safety. It is not intended to review the results obtained by other cardiologists, because wide differences in the way that the techniques are practised make comparisons difficult, and most published reports have described small series.

\section{SuBJECTS AND METHODS}

The patients' ages lay between 5 and 72 years. Of the 508 patients, 376 had mitral valvar disease with or without coincidental aortic lesion; 84 had aortic valvar disease, and 48 had a variety of congenital and acquired heart diseases. The majority of the mitral patients had systolic murmurs or a previous heart operation, and the investigation was done to assess the relative importance of various factors: stenosis, re-stenosis, regurgitation, "the myocardial factor", coincidental aortic disease, or pulmonary vascular changes.

Acting under the firm belief that tension and fear predispose the subject of left heart catheterization to complications, great attention was paid to the mental preparation of the patient. Before the investigation he was made familiar with the catheterization laboratory where, from choice, the preliminary clinical examination and heart-sound recordings were carried out; and he came to know the various doctors, nurses, and technicians. The procedures that would require the patient's co-operation were carefully explained and rehearsed beforehand. A quiet demeanour, equanimity, and competence were required from the laboratory staff. Tachycardia and dyspnea prevent the recording of diagnostic tracings, and, if necessary, investigation was deferred until medical treatment abolished tachycardia, allowed the patient to hold his breath without difficulty for at least 20 seconds, and enabled him to lie flat, or nearly flat, without discomfort.

A highly penetrated postero-anterior teleradiogram of the chest (Fig. 1) was taken to show the relation of the left atrial shadow to the right heart border of the spine; and to allow measurement of the left atrial area, because enlargement of the left atrium modified the physiological 


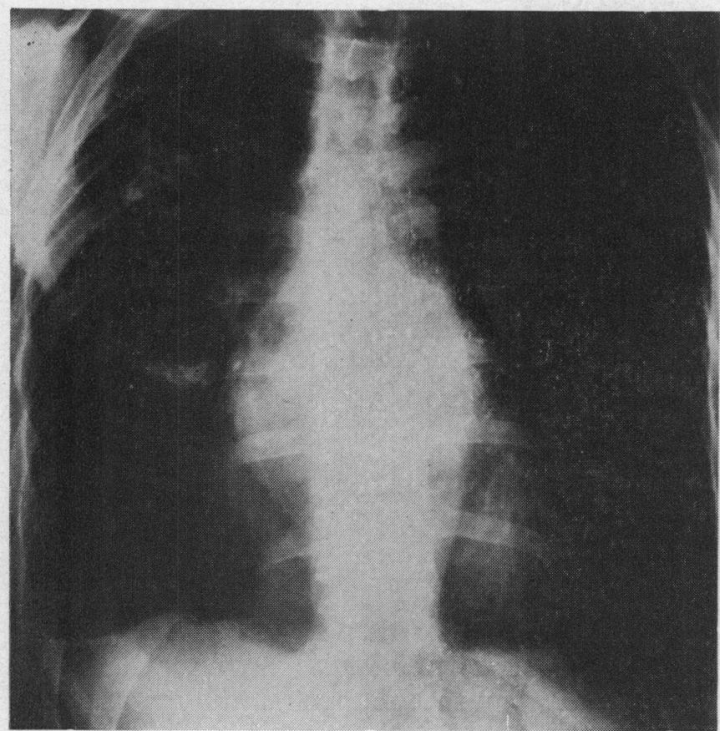

Fig. 1.-Penetrated postero-anterior chest film showing the size and shape of the left atrium.

features of mitral disease (Nixon and Snow, 1962; Nixon and Ikram, 1963).

The patient was premedicated with pentobarbitone $120 \mathrm{mg}$. two hours before the start of the investigation, and omnopon $10 \mathrm{mg}$. and hyoscine $0.2 \mathrm{mg}$. one hour beforehand, unless the body weight or the liver condition required the dose to be modified. The laboratory equipment was tested before the patient arrived. During the investigation noise and conversation were reduced to a minimum, and the patient was warned before he was touched or disturbed. This management, together with the preparation, usually produced a tranquil drowsy condition, with two to three hours of relative freedom from fluctuation of the oxygen consumption, cardiac output, and cardiovascular pressure pulses.

With the patient supine on a comfortable mattress on the fluoroscopy table, and under generous local anaesthesia, an indwelling needle was inserted into the brachial artery, or a catheter was passed into the aorta after percutaneous insertion into the femoral artery. The right long saphenous vein was localized by palpation through a $2 \mathrm{~cm}$. incision into the groin; it was usually patent even when it had been tied with catgut during a previous investigation or varicose vein operation. In some 70 cases the left vein was used in order to leave the right groin intact for the subsequent open-heart operation. Occasionally the Seldinger technique (Seldinger, 1953; Brockenbrough and Braunwald, 1960) was used to introduce the septal puncture instruments into the femoral vein.

After the preliminary right-heart catheterization the Ross (Ross, 1959) or the Brockenbrough (Brockenbrough and Braunwald, 1960) method of transseptal left heart catheterization was performed, and in certain cases, percutaneous left ventricular puncture.

\section{The Ross Technique}

A $59.5 \mathrm{~cm}$. Cournand type catheter with a detachable hub is passed to the right atrium from the groin and then a $61 \mathrm{~cm}$. 17-gauge Ross needle (Fig. 2) is inserted into the catheter and advanced until its point is just concealed by the tip of the catheter. The fossa ovalis is found by palpation under fluoroscopic control, and the catheter tip and needle point are bedded down in it with firm pressure. The floor of the fossa is punctured by advancing the needle point $1.2 \mathrm{~cm}$. beyond the catheter tip in a medial and posterior direction. The needle point is recognized as being in a satisfactory position when oxygenated blood can be withdrawn freely and an undistorted left atrial pressure pulse can be recorded through the needle. A Clay-Adams PE 50 catheter (Fig. 2), $90 \mathrm{~cm}$. in length, is then introduced through the needle into the left atrium, and under certain circumstances into the left ventricle and even the aorta. The PE 50 catheter passes from atrium to ventricle most easily when the curvature of the needle is held in the coronal plane.

Comments on the Ross Technique. Force should never be used to insert the needle through the catheter; if there is difficulty in negotiating the curve of the pelvis the needle and catheter should be advanced together under fluoroscopic control, and then the catheter should be drawn back over the needle. It is more dangerous to introduce the Ross needle through a teflon catheter with a smooth lining than through a Cournand type catheter, because the latter has a woven lining which causes a fine thrill as the needle point moves down the lumen; the thrill ceases if the moving needle point perforates the wall of the catheter. The rigidity of the Ross needle is an advantage in the palpation and puncturing of the fossa ovalis.

The site of the fossa ovalis is remarkably constant whether or not the left atrial chamber is greatly enlarged. It is rare for the fossa to lie anywhere but over the right margin of the spine some $2-3 \mathrm{~cm}$. above the point where the left atrial shadow crosses the spine. In cases with small left atria the fossa is concave and easier to palpate than it is when great left atrial enlargement has made its floor convex. In many cases with small left atria the catheter tip and needle pass through a slit foramen ovale during the initial palpation, and if this is not appreciated, the root of the aorta or the posterior wall of the left atrium may be punctured when the needle point is advanced.

If the PE 50 catheter becomes knotted in the left atrium and cannot be pulled back through the needle, it is a simple matter to withdraw the needle and to pull out the knotted PE 50 catheter through the short No. 9 catheter.

One patient died from a complication of surgical treatment 10 days after septal puncture, and at necropsy it was scarcely possible to see the slit caused by the needle puncture.

The atrial septum pulsates a little with every heart beat, but it scarcely moves during normal breathing, and the Ross needle may be left in situ for one or two hours without causing discomfort. This removes any sense of 


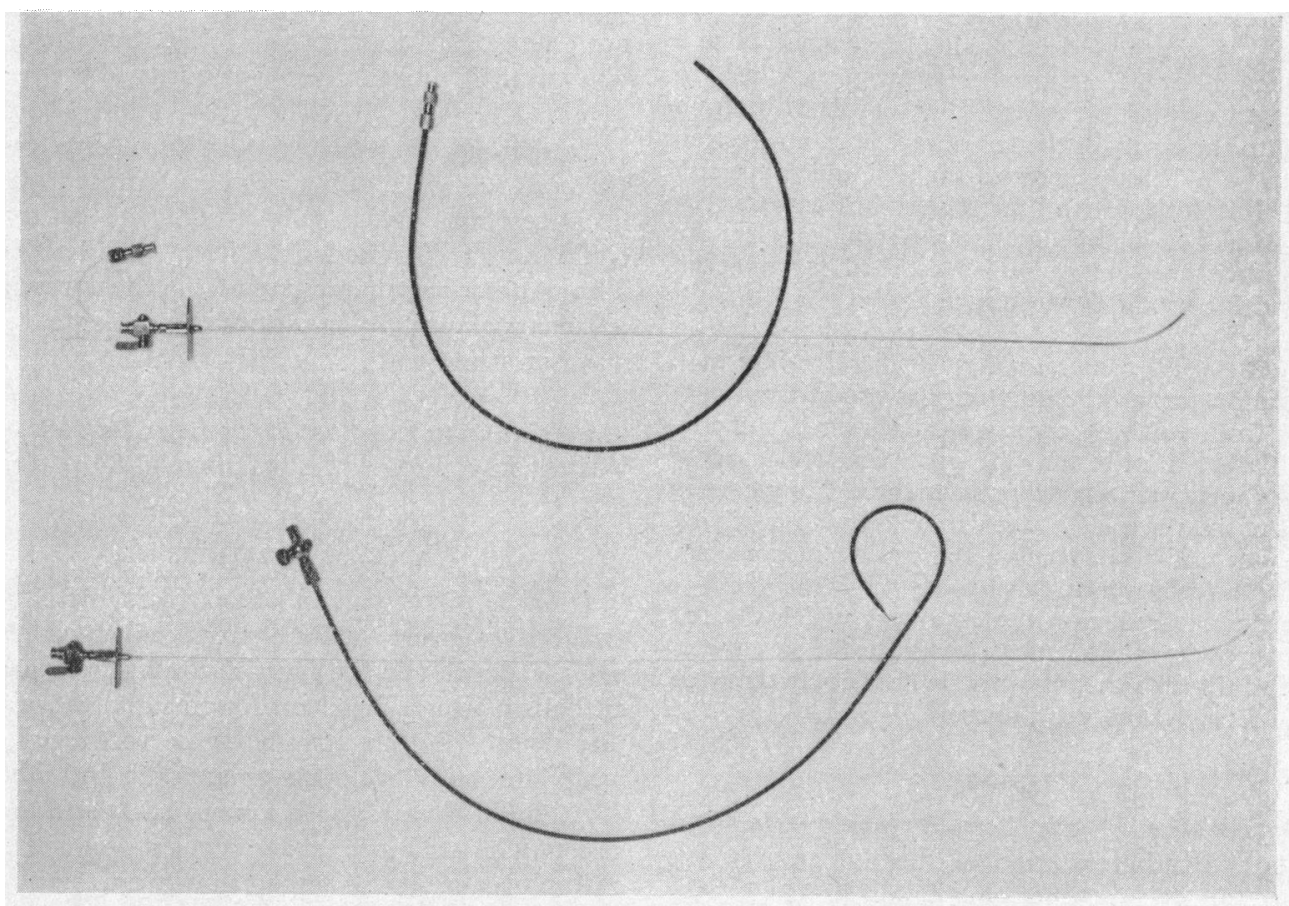

FIG. 2.-Above: Ross transseptal needle and outer catheter. A PE 50 catheter is shown emerging from the needle. Below: the Brockenbrough transseptal needle and outer catheter.

urgency from the investigation, and gives ample time for the collection and development of photographic records.

\section{The Brockenbrough TechniQue}

The needle employed in this modification of the Ross technique is longer and thinner than the Ross needle. When the fossa has been punctured the catheter carrying the needle is advanced over it into the left atrium, and manipulated under fluoroscopic control into the left ventricle. The relatively large size of the catheter introduced into the left side of the heart permits the injection of contrast medium for angiocardiography. Its construction allows it to be inserted into the femoral vein by the Seldinger technique, thus eliminating the need to expose the long saphenous vein.

Comments on the Brockenbrough Technique. Our methods of diagnosing acquired heart disease have infrequently made use of angiocardiography, and so the chief indication for the Brockenbrough modification has been the need to introduce the instruments directly into the femoral vein. The Brockenbrough needle, being longer and thinner than the Ross needle, is correspondingly less efficient as an instrument for palpating the inside of the heart. The advance of the needle point is difficult to control, and the pericardium or the aorta may easily be perforated. Even when the puncture of the fossa is achieved, it may be difficult to pass the catheter over the needle into the left atrium. The catheter is large and stiff and may perforate the left atrial appendage. The hole it makes in the atrial septum is larger and more ragged than the Ross needle puncture, and fatal embolization has resulted from clot formation at its site (Pinkerson, Kelser, and Adkins, 1963). The large bore of the Brockenbrough catheter permits pressure records to be obtained with pressure transducers of poorer quality than those required by the PE 50 catheter, but it is inexcusable to choose the more difficult and dangerous technique merely because the laboratory is inadequately equipped. It is advisable not to practise the Brockenbrough technique until confidence has been gained by the use of the easier Ross technique. Care should be taken to choose a needle of the type used by Brockenbrough (manufactured by Becton-Dickinson) and to avoid the inferior substitutes that have been sold.

\section{Percutaneous Left Ventricular Puncture}

The method of percutaneors puncture of the apex of the left ventricle is derived from Brock, Milstein, and Ross (1956), but the fact that we have chiefly used it to obtain the left ventricular pressure pulse in mitral regurgitation, rather than to assess cases of aortic stenosis, has permitted certain modifications.

The puncture is only performed when the outward movement of the apex of the left ventricle in systole is palpable through the chest wall. The skin and intercostal tissues are generously infiltrated with local anaesthetic; the equipment monitoring the left atrial and 
arterial pressures is checked; the transducer for the left ventricular pressure is made ready, and connected to a saline-filled 20 or 21 standard wire guage needle by a short length of plastic tubing. The needle is inserted swiftly into the cavity of the left ventricle in a direction judged to be $45^{\circ}$ to the coronal, sagittal, and transverse planes. It is flushed with $1 \mathrm{ml}$. saline; the electrocardiogram is inspected for freedom from ectopic activity; the left ventricle pressure pulse is recorded for a dozen beats, and the needle is withdrawn.

Comments on Percutaneous Left Ventricular Puncture. Complications usually result from the use of large bore needles; from stabbing the needle backwards and forwards in an attempt to find the small ventricular cavity in cases of aortic stenosis; from using the technique when the apex of the ventricle is impalpable through overlying lung tissue; and from leaving the needle in place for more than a few seconds.

In aortic valvar disease the ease with which the left ventricular cavity may be entered with the PE 50 catheter introduced through the Ross needle has largely removed the need for percutaneous puncture.

\section{RESULTS}

Catheterization of the Left Atrium by the Ross Technique. Catheterization of the left atrium was attempted in 467 patients, and failed in $16(3.4 \%)$. The reasons for failure were as follows. The lack of suitable veins (4 cases); gross kyphoscoliosis (2 cases); acute respiratory distress ( 1 case); inability to pierce a surgically plicated septum (3 cases); dysrhythmia on palpation of the septum (1 case); and failure to accomplish needle puncture of the fossa ovalis from lack of experience or inadequate intensity of fluoroscopic illumination (5 cases).

Catheterization of the Left Ventricle by the Ross Technique. Passage of the PE catheter into the left ventricle was attempted in 83 cases of aortic valvar disease with 13 failures $(16 \%)$; in 62 cases of mitral obstruction and regurgitation with 51 failures $(82 \%)$; and in 99 cases of mitral incompetence with 76 failures $(76 \%)$. A determined effort was made to reach the left ventricle in the cases of aortic valvar disease, and failure to do so in the majority of cases was due to the use of plastic catheters which were not as satisfactory as the PE 50 .

Catheterization of the Left Atrium by the Brockenbrough Technique. Attempts were made to reach the left atrium in 62 cases, and failed in $5(8 \%)$. The reasons for failure were as follows. The needle perforated the catheter ( 1 case); the needle perforated the pericardium ( 2 cases); and surgical plication of the septum ( 2 cases).

Catheterization of the Left Ventricle by the
Brockenbrough Technique. The left ventricle was not entered in 38 out of $57(67 \%)$, but the attempt was seriously made only in a minority of the patients.

Retrograde Catheterization of the Aorta from the Femoral Artery. This was attempted in 115 instances, and failed in 4 where the catheter tip could not be persuaded to leave the iliac vessels. The catheter was manœuvred from the aorta into the left ventricle in 35 per cent of the patients, but this was not attempted in the majority.

Percutaneous Puncture of the Left Ventricle. This was accomplished without failure in 84 patients.

\section{COMPLICATIONS}

Deaths. One woman of 60 years, with chronic congestive heart failure from advanced mitral disease, developed the signs of coronary and cerebral embolism after a Brockenbrough catheter was introduced into the left atrium: these recovered, but a week later she developed pneumonia and died.

A boy of 5 years, with a huge heart and diureticresistant heart failure, underwent a prolonged investigation because it was hoped that a remediable lesion might be found. Shunts, and right- and left-sided valvar lesions were excluded. He became anoxic and died from ventricular fibrillation before tracheotomy could be performed. Necropsy revealed gross idiopathic hypertrophy of the heart.

These two deaths $(0.4 \%)$ occurred in 508 investigations in which transseptal catheterization was employed. The embolization complicating the first case was attributable to the catheterization of the left atrium, but the death of the second case was not related to it.

Acute Bleeding Tendency. Two patients developed an acute severe bleeding diathesis for several hours after left heart catheterization. In one, this resulted in a severe hæmatoma of the arm from bleeding at the Cournand needle puncture site. In the other a massive hæmothorax and hæmopericardium followed percutaneous left ventricular puncture and required surgical drainage. The cause of the failure to clot was not recognized. Heparin overdose was considered unlikely.

Air Embolism. A transient hemiplegia in two patients, with complete recovery, was attributed to the accidental injection of small amounts of air into the left atrium. In one of them the air also entered a coronary artery and caused a temporary cardiac arrest which was successfully treated by closed massage. 
Dysrhythmia. Septal puncture appeared to precipitate atrial fibrillation in one patient, and flutter in three.

Pallor, Hypotension, Sweating, and Bradycardia. These signs caused the investigation to be terminated in 11 patients, but they recovered quickly without any permanent untoward effect.

Perforation of the Pericardium. This occurred in 1 out of 467 attempts to puncture the septum by the Ross technique, and in 3 out of the 62 attempts by the Brockenbrough method. In one of the latter it was necessary to drain a hæmopericardium; in the remainder the accident caused no ill effect.

Local Arterial Thrombosis. Femoral arterial obstruction occurred at the site of introduction of the Seldinger catheter in 2 patients, and in one of these the clot had to be removed by operation.

Comment on the Complications. These undesirable complications occurred in $23(4.5 \%)$ out of a total of 508 patients undergoing left heart catheterization. The method of catheterization was irrelevant to the cause of one of the two deaths; and accidental air embolism in a patient was the one serious complication arising from the use of the Ross technique in 467. This complication is unlikely to occur when image intensification allows an adequate amount of light to be used in the catheterization laboratory.

\section{Discussion}

From the point of view of safety there can now be little doubt that the Ross technique has much to commend it. The one death in 467 attempts resulted from ventricular fibrillation complicating respiratory failure in a desperately ill child, and was unrelated to the use of the needle. Three patients developed a remediable supraventricular tachycardia; eight had transient pallor, hypotension, and bradycardia, and one patient suffered no discomfort from accidental puncture of the pericardium. One patient recovered completely from cardiac arrest caused by accidental air embolism. This is a serious hazard of left heart catheterization, and may be eliminated when the use of an image intensifier permits a better illumination of the catheterization room.

From the patient's point of view the painless investigation allowed an accuracy of diagnosis which eliminated fruitless thoracotomies and needless exclusion from surgical treatment.

From the physician's point of view the investigations removed doubt from the diagnosis before operation, at a time when the interpretation of many of the physical signs of mitral and aortic disease was erroneous or open to dispute. Comparison of the physical signs with the left heart catheter records, and the surgeon's observation, has clarified their interpretation in the minds of the authors, and the findings have been published (Nixon and Wooler, 1961 ; Nixon, 1961a, b; Nixon and Wooler, 1963a, b; Nixon, 1963; Nixon and Ikram, 1963; Nixon, Hepburn, and Ikram, 1964; Ikram, Nixon, and Fox, 1964).

This improved understanding of the physiological import of the physical signs has led the authors now to prefer phonocardiography and cardiac-impulse recording to left heart catheterization in the majority of patients with acquired heart disease who consequently no longer require admission for investigation (Nixon and Wooler, 1963a, b; Nixon and Ikram, 1963; Nixon et al., 1964; Nixon, 1964).

Brockenbrough's technique is more difficult to master than Ross's, and is more dangerous in our hands. The dangers have been increased in this country by the sale of poorly constructed needles.

Retrograde catheterization of the aorta from the femoral artery was performed in 111 patients, and caused femoral arterial obstruction in 2: in 1, clot had to be removed at operation; and in the other the signs of obstruction resolved spontaneously and there were no clinically detectable after effects. Entry into the left ventricle from the aorta was rarely attempted because image intensification was not available to the authors.

An indwelling Cournand needle was introduced into the brachial artery in 389 patients. Afterwards some complained of pain or a small hæmatoma, but the discomfort was not serious or prolonged. After the investigation one patient developed an acute bleeding tendency which resulted in a large hæmatoma around the brachial arterial wound.

Percutaneous left ventricular puncture was easily performed in 84 patients, in one of whom an acute bleeding tendency caused hæmothorax and hæmopericardium.

The choice of patient and method for entry into the left ventricle has crystallized in our minds. Contrary to popular opinion it is rarely necessary in mitral disease because the important physiological feature, the shape and duration of the left ventricular filling pressure gradient, may usually be obtained from the configuration and duration of the mitral diastolic murmur (Fig. 3) (Nixon and Wooler, 1963a, b). The size of the mitral orifice and the choice of operation in cases with regurgitation can be assessed without entering the left ventricle (Nixon and Wooler, 1961; Nixon, 1961a; Nixon and Wooler, 1963a, b; Nixon, 1964). Where aortic valvar disease complicates a mitral lesion, the left ventricular pressure pulse may so often be obtained by the 


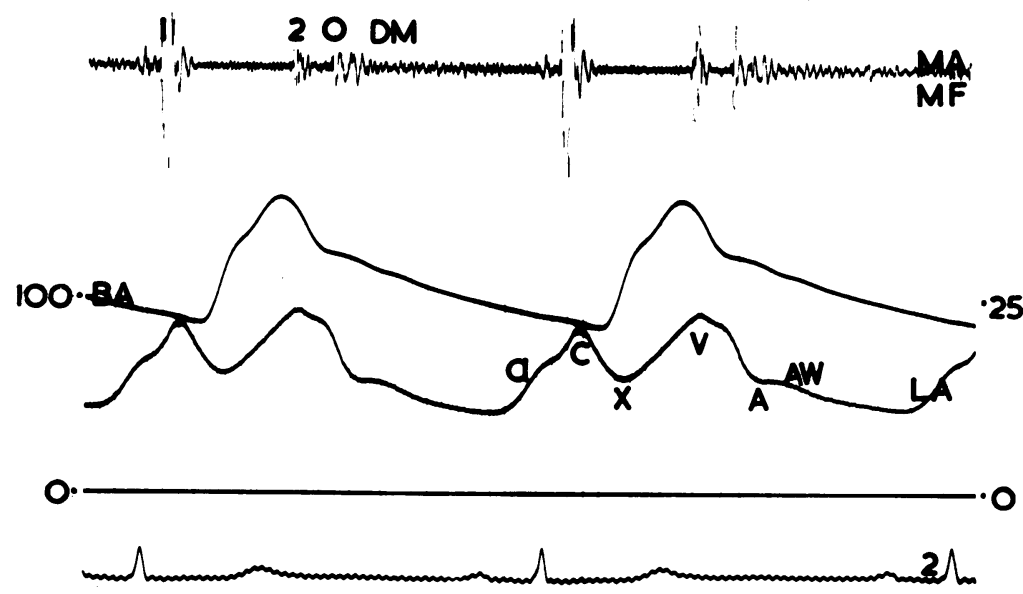

FIG. 3.-Left atrial pressure pulse (LA) recorded through PE 50 catheter within a Ross needle, in mitral stenosis.

safe Ross technique that it is worth while to use it as the first step. If it fails, the Brockenbrough method, retrograde catheterization from the aorta, or percutaneous puncture may be used. When employed in the circumstances described above percutaneous puncture is simple, effective, and safe. In cases of

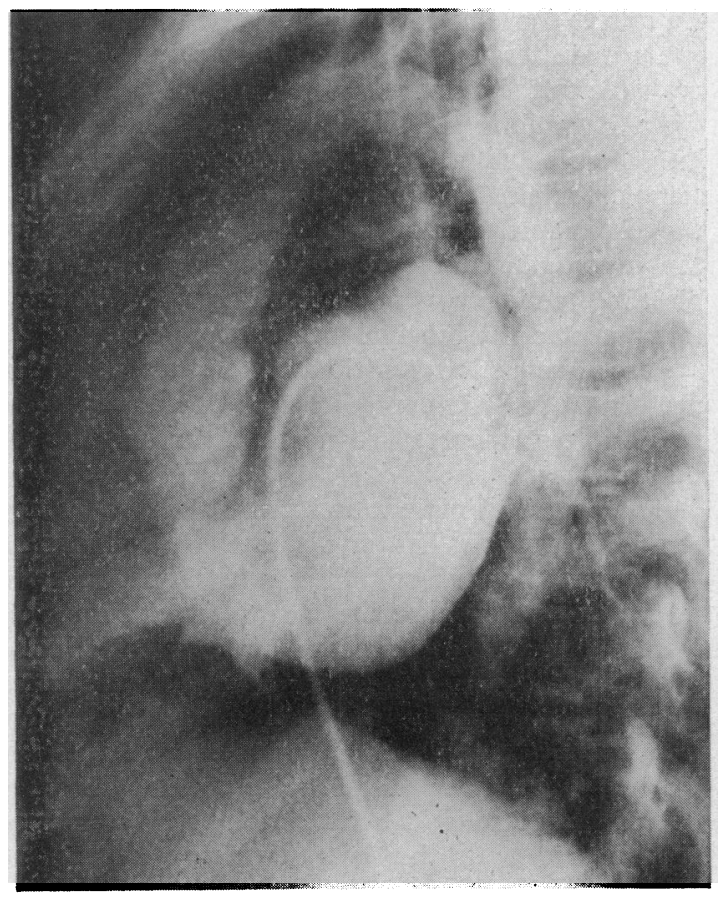

Fig. 4.-Left atrial angiocardiogram through Brockenbrough catheter outlining details of the left ventricle in a case of obstructive cardiomyopathy. obstruction to outflow from the left ventricle, e.g. in aortic stenosis, we fear the dangers of percutaneous left ventricular puncture and rely upon the ease and sureness with which it is possible to enter the left ventricle through a normal mitral valve by one of the transseptal techniques. In aortic regurgitation there is little difficulty in entering the left ventricle from the aorta, and sometimes the catheter tip may be manipulated into the left atrium.

The question of the choice of the patient and the method for selective left ventricular angiography is raised frequently enough to deserve comment. We believe that it is rarely necessary. In mitral regurgitation, a phonocardiogram and a record of the impulse from the skin overlying the apex of the left ventricle, supplemented if necessary by a left atrial pressure pulse and a blue dye curve (Nixon and Snow, 1962), provide more useful diagnostic information with less risk. The exceptional patients who may require left ventricular angiocardiography are those who have been subjected to a plastic repair of the mitral valve, or to the insertion of a prosthetic mitral valve.

In the various forms of congenital and acquired obstruction to outflow from the left ventricle, where angiocardiography has been desirable, we have been satisfied with the results of the easier and safer injection of opaque medium into the left atrium through the Brockenbrough catheter (Fig. 4), or into the root of the aorta (catheterized by the Seldinger technique from the femoral artery). The left atrium has been a satisfactory injection site for the angiocardiographic demonstration of coarctation of the aorta.

In our experience it has not been worth while to attempt to record the indirect left atrial pulse by 
wedging a catheter in a branch of the pulmonary artery when a direct left atrial pulse has been obtainable by the Ross technique. One reason is that the wedged pressure can only be relied upon for diagnosis when its height and shape have been verified by comparison with the direct pulse. Another reason is the fact that the attempt usually is unsuccessful where pulmonary hypertension complicates severe left heart disease. The other reasons are the frequent and variable distortion in shape, and delay in time of the wedged pulse; and its usual failure to show the stasis wave, a feature of the direct left atrial pulse that distinguishes the patients with relatively large mitral orifices from those with small orifices who require valvotomy for the relief of obstruction.

\section{SUMmaRY AND CoNClusions}

The authors performed left heart catheterization in 508 patients during a period of six years. Needle puncture of the atrial septum was employed in every case and was supplemented by percutaneous catheterization of the aorta and/or percutaneous left ventricular puncture where necessary.

The important factors relating to the safety of the techniques, and the complications, are described in detail. The evidence suggests that the Ross technique may be considered safe enough for use outside thoracic surgical centres when diagnosis requires entry into the left atrium. This is the method of choice when the left ventricular pressure pulse is required in aortic valvar disease, and in other conditions where there is freedom from the extreme forms of mitral obstruction and incompetence. Mitral valvar disease rarely calls for entry into the left ventricle, because the configuration and duration of the mitral diastolic murmur, the pulsation of the skin overlying the apex of the left ventricle, and the presence or absence of the left atrial stasis wave, indicate the size of the mitral orifice and the severity of the regurgitation. Left ventricular angiography is rarely needed because the important information can usually be obtained by injecting the radioopaque medium into the left atrium or the root of the aorta. The left atrium is an injection site that may be used with advantage in coarctation of the aorta. The Brockenbrough technique appears to be more difficult and dangerous than the Ross, particularly when poorly constructed instruments are used, and the authors believe that it should not be used until the operator is practised in the Ross technique. Percutaneous left ventricular puncture, with the above-mentioned provisos, appears to be safe and successful.

The authors have abandoned the use of the wedged pulmonary arterial pulse as a substitute for the direct left atrial pulse.

The physiological information obtained by the authors has allowed them increasingly to rely upon the out-patient technique of phonocardiography and cardiac impulse recording for objective diagnosis in the majority of patients with disease of the left side of the heart.

The authors wish to express their gratitude to Dr. John Ross jun. and Dr. Eugene Braunwald for teaching one of them the transseptal technique. They are also deeply indebted to Mr. G. H. Wooler and Professor R. E. Tunbridge for making this study possible. They are grateful to Mr. H. M. Snow, Mr. R. Addyman, Mr. H. Ketteringham, and the Charing Cross Hospital Research Laboratories for assistance. The Board of Governors of the United Leeds Hospitals, the Trustees of the Nuffield Foundation, and private benefactors have provided financial help.

\section{REFERENCES}

Brock, R., Milstein, B. B., and Ross, D. N. (1956). Percutaneous left ventricular puncture in the assessment of aortic stenosis. Thorax, 11, 163.

Brockenbrough, E. C., and Braunwald, E. (1960). A new technic for left ventricular angiography and transseptal left heart catheterization. Amer. F. Cardiol., 6, 1062.

Ikram, H., Nixon, P. G. F., and Fox, J. A. (1964). The haemodynamic implications of the bisferiens pulse. Brit. Heart f., 26, 452.

Lancet (1957). Annotation. Left-heart catheterisation. 2, 630.

Nixon, P. G. F. (1960). The transseptal approach to the left atrium in mitral regurgitation. Thorax, 15, 225.

(1961a). The third heart sound in mitral regurgitation. Brit. Heart F., 23, 677.

(1961b). Time relationships of the left atrial $\mathrm{V}$ wave in mitral valvular disease. Brit. Heart f., 23, 637.

(1963). The genesis of the third heart sound. Amer. Heart $\mathcal{F}$., 65, 712 .

(1964). The diagnosis of the mitral lesion in patients with regurgitation. Postgrad. med. F., 40, 136.

- Hepburn, F., and Ikram, H. (1964). Simultaneous recording of heart pulses and sounds. Brit. med.f., 1, 1169.

— patients with giant left atria. Thorax, 18, 307.

- and Snow, H. M. (1962). Indicator dilution curves in mitral valvular disease. Brit. Heart. F., 24, 637.

- , and Wooler, G. H. (1961). Rapid left ventricular filling and stasis in mitral regurgitation. Brit. Heart f., 23, 161.

$\longrightarrow$, and - (1963a). Left ventricular filling pressure gradient in mitral incompetence. Brit. Heart $7 ., 25,382$. and - (1963b). Phases of diastole in various syndromes of mitral valvular disease. Brit. Heart $\mathcal{F}$., 25, 393.

Pinkerton, A. L., Kelser, G. A., Jr., and Adkins, P. C. (1963). Mural thrombus in the left atrium secondary to transseptal catheterization of the left side of the heart. New Engl. F. Med., 268, 367.

Seldinger, S. I. (1953). Catheter replacement of the needle in percutaneous arteriography. A new technique. Acta radiol. (Stockh.), 39, 368.

Ross, J., Jr. (1959). Transeptal left heart catheterization: A new method of left atrial puncture. Ann. Surg., 149, 395. 